\title{
Characterization and Comparison of Detector Systems for Large Area X-ray Imag- ing
}

Jeffrey M. Davis ${ }^{1, *}$, Julia Schmidt ${ }^{2}$, Martin Huth $^{2}$, Sebastian Ihle ${ }^{2}$, Daniel Steigenhöfer ${ }^{2}$, Peter Holl ${ }^{2}$, Gerhard Lutz ${ }^{2}$, Udo Weber ${ }^{1}$, Adrian Niculae ${ }^{1}$, Heike Soltau ${ }^{1}$, Lothar Strüder ${ }^{2}$

1. PNDetector GmbH, Otto-Hahn-Ring 6, 81739 München, Germany

2. PNSensor GmbH, Otto-Hahn-Ring 6, 81739 München, Germany

In the last 15 years, there has been a major shift in the type of detector used in X-ray microanalysis [1]. The silicon drift detector (SDD) is now the predominant energy dispersive X-ray spectrometer, having replaced the lithium drifted silicon detector. With high spectral resolution at input count rates over 100000 counts/s, an SDD reduces the total time required to record a high quality X-ray image. In 2005, a multi-element SDD was also introduced, which combined multiple SDDs into a single detector [2]. These detectors combine large solid angles (for more efficient X-ray collection) with fast spectral processing through multiple pulse processors. Yet its primary drawback is that the SDD does not record the position of each X-ray event in addition to the energy. An array of SDDs could do this, but the position resolution would be poor. Otherwise, an X-ray scintillator combined with an ordinary charge coupled device (CCD) can record the location of the X-ray event, but the energy resolution is usually poor. An ideal imaging spectrometer would be one that can record both position and energy.

In 2011, PNSensor introduced an X-ray pnCCD camera, an energy dispersive X-ray spectrometer developed in collaboration with PNDetector. The pnCCD is a unique CCD that combines the excellent position resolution of the CCD with the energy resolution of an SDD. The pnCCD is able to collect X-rays at high count rates with the spectral resolution of every pixel on par with high performing SDDs, because it records both the position of the X-ray event and the energy. This spectroscopic imaging capability opens up new opportunities for X-ray imaging and analysis, especially for large samples analyzed in the $\mu \mathrm{XRF}$.

Numerous experiments have been performed to test and quantify the spectroscopic qualities of the detector as they relate to high precision X-ray microanalysis. Functioning purely as a spectrometer, the pnCCD can count at a rate of 200000 counts/s (or more) with a resolution of approximately $150 \mathrm{eV}$ at Mn K-alpha. However, when a polycapillary optic is placed in front of the pnCCD to focus the incoming X-rays, the pnCCD can be used to produce X-ray images. This is particularly useful in $\mu$ XRF where the pnCCD eliminates the requirement of scanning the sample under the X-ray beam by recording all 70000 spectra at once. The imaging area of the pnCCD is $1.2 \mathrm{~cm}$ x $1.2 \mathrm{~cm}$, with a pixel size of $48 \mu \mathrm{m}$ (sub pixel resolution down to $10 \mu \mathrm{m}$ possible). Although the electronics and data processing are different from the SDD, the end result of an experiment performed with the pnCCD is an X-ray Spectrum Image (a full X-ray spectrum stored at each pixel). As a large area imaging spectrometer, the pnCCD has proved extremely useful in diverse fields such as cultural heritage conservation and materials science, where efficient, large area imaging is necessary [4]. This work is primarily concerned with the advantages and opportunities provided by the pnCCD when used as an imaging spectrometer in a $\mu$ XRF system. As shown in Figure 1, the spectral performance of the detector, while counting at a rate of over 200000 counts/s is easily comparable to an SDD counting at a rate of approximately 20000 counts/s. Figure 2 shows an image produced with the pnCCD. The image was collected in approximately $600 \mathrm{~s}$, and it covers an area of $1.4 \mathrm{~cm}^{2}$. To get the same data from an SDD based system, the stages in a conventional $\mu \mathrm{XRF}$ would have to move at a rate of $5 \mathrm{~m} / \mathrm{s}$ 
and count at a rate of 115000 counts/s.

References:

[1] Strüder, L., Fiorini, C., Gatti, E., et al, High resolution non dispersive x-ray spectroscopy with state of the art silicon detectors, Mikrochimica Acta, Supplement 15 (1998) 11

[2] Longoni, A., Fiorini, C., Guazzoni, C., et al, A novel high-resolution XRF spectrometer for elemental mapping based on a monolithic array of silicon drift detectors and on a polycapillary X-ray lens, X-ray Spectrometry, 34 (2005), p. 439:445.

[3] Scharf, O., Ihle, S., Ordavo, I., et al, Compact pnCCD-nased X-ray camera with high spatial and energy resolution: A color X-ray camera, Analytical Chemistry, 83 (2011), p. 2532:2538.

[4] Romano, F.P., Caliri, C, Cosentino, L., et al, Macro and micro full field X-ray fluorescence with an X-ray pinhole camera presenting high energy and high spatial resolution, Analytical Chemistry, 86 (2014), p.10892:10899.

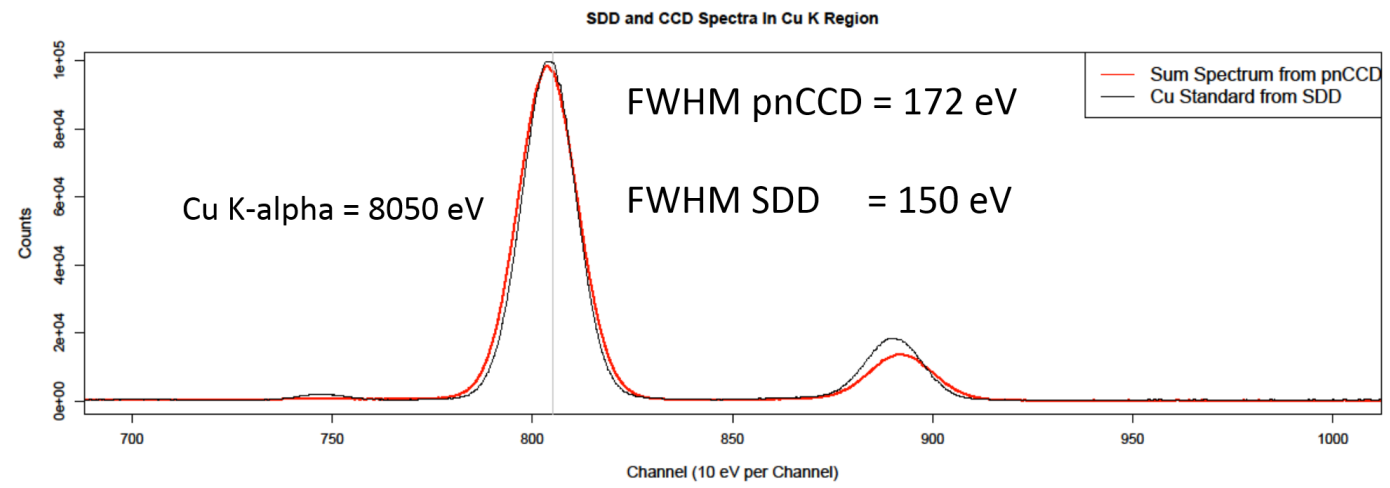

Figure 1: Spectral comparison of a spectrum from an SDD (black line) and a spectrum made with a pnCCD (red line). The pnCCD spectrum has an equivalent resolution of $152 \mathrm{eV}$ at Mn K-alpha The performance of the spectrometer at a count rate of over 200000 counts/s is very good for imaging purposes.

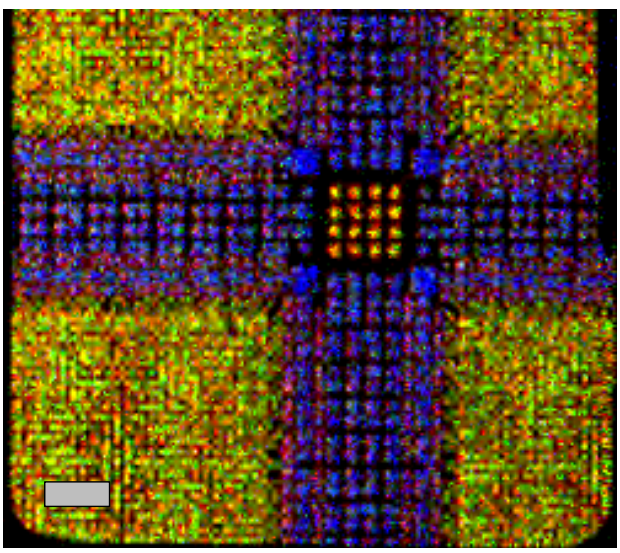

Figure 2: The X-ray image of a section of an IBM circuit board has dimensions of $1.2 \mathrm{~cm} \mathrm{x} 1.2 \mathrm{~cm}$, and it was acquired in $600 \mathrm{~s}$. Yet this image was produced with the pnCCD without moving the stage, fully resolving spatial features, such as the wires in the center, which are approximately $150 \mu \mathrm{m}$ in diameter. Here the $\mathrm{Cu} \mathrm{X}$-rays are colored in red, the Au X-rays are in blue and the Ni X-rays are in green. The scale bar represents $1.4 \mathrm{~mm}$ on the image. 